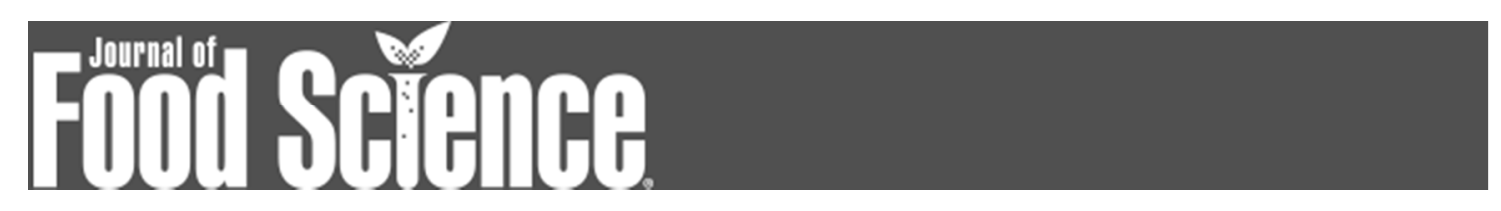

\title{
Macromolecular traits in the African rice Oryza glaberrima and in glaberrima/sativa crosses, and their relevance to processing
}

\begin{tabular}{|r|l|}
\hline Journal: & Journal of Food Science \\
\hline Manuscript ID & JFDS-2017-0564.R1 \\
\hline Danuscript Type: & Food Chemistry \\
\hline Complete List of Authors: & \begin{tabular}{rl|} 
Marengo, Mauro; Università degli Studi di Milano, DeFENS \\
Barbiroli, Alberto; University of Milan, Milan, Italy, DeFENS \\
Bonomi, Francesco; University of Milan, Milan, Italy, DeFENS \\
Casiraghi, Cristina; University of Milan, DeFENS \\
Marti, Alessandra; Università degli Studi di Milano, DeFENS \\
Pagani, Maria Ambrogina; Università degli Studi di Milano, DeFENS \\
Manful, John; Africa Rice Center \\
Graham-Acquaah, Seth; Africa Rice Center \\
Ragg, Enzio; University of Milan, Milan, Italy, DeFENS \\
Fessas, Dimitrios; Università degli Studi di Milano, DeFENS \\
Hoogheboom, John; Università degli Studi di Milano, DeFENS \\
Iametti, Stefania; DeFENS
\end{tabular} \\
\hline Keywords: & Oryza glaberrima, Oryza sativa, starch, proteins, rice \\
\hline \multicolumn{2}{|l}{} \\
\hline
\end{tabular}

\section{SCHOLARONE \\ Manuscripts}


3

4

5

6

7

\section{Macromolecular traits in the African rice Oryza glaberrima and in glaberrima/sativa crosses,} and their relevance to processing

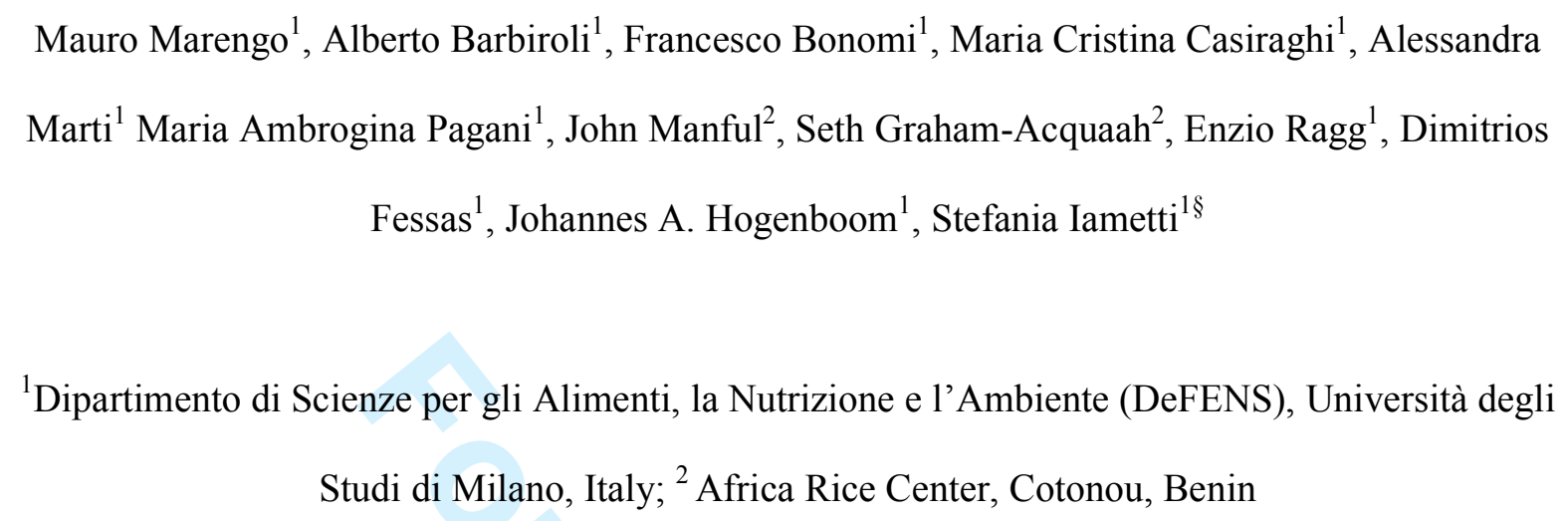

${ }^{1}$ Dipartimento di Scienze per gli Alimenti, la Nutrizione e l'Ambiente (DeFENS), Università degli Studi di Milano, Italy; ${ }^{2}$ Africa Rice Center, Cotonou, Benin

${ }^{\S}$ Corresponding author

stefania.iametti@unimi.it

DeFENS

Via G. Celoria, 2

20133 Milan, Italy

Phone $+3902-5031-6819$

Fax +3902-5031-6801

Word count: 5688

Short version of the title:

\section{Molecular traits of Oryza glaberrima}




\section{Abstract}

Molecular properties of proteins and starch were investigated in two accessions of Oryza glaberrima and Oryza sativa, and in one NERICA cross between the two species, to assess traits that could be relevant to transformation into specific foods. Protein nature and organization in $O$. glaberrima were different from those in $O$. sativa and in NERICA. Despite the similar cysteine content in all samples, thiol accessibility in $O$. glaberrima proteins was higher than in NERICA or in $O$. sativa. Inter-protein disulphide bonds were important for the formation of protein aggregates in O. glaberrima, whereas non-covalent protein-protein interactions were relevant in NERICA and O. sativa. DSC and NMR studies indicated only minor differences in the structure of starch in these species, as also made evident by their microstructural features. Nevertheless, starch gelatinization in O. glaberrima was very different from what was observed in $O$. sativa and NERICA. The content of soluble species in gelatinized starch from the various species in the presence/absence of treatments with specific enzymes indicated that release of small starch breakdown products was lowest in $O$. glaberrima, in particular from the amylopectin component. These findings may explain the low glycaemic index of $O$. glaberrima, and provide a rationale for extending the use of $O$. glaberrima in the production of specific rice-based products, thus improving the economic value and the market appeal of African crops.

Practical Application: The structural features of proteins and starch in O. glaberrima are very different from those in $O$. sativa and in the NERICA cross. These results appear useful as for extending the use of $O$. glaberrima cultivars in the design and production of specific rice-based products (e.g., pasta), that might, in turn, improve the economic value and the market appeal of locally sourced raw materials, by introducing added-value products on the African market. 


\section{Introduction}

Rice is one of the main crops cultivated worldwide, and is a primary source of food for more than two-thirds of the world's population (Singh and others 2013; Gayin and others 2015a). The genus Oryza comprises two distinct types of domesticated rice: Oryza sativa (Asian rice) and Oryza glaberrima (African rice). Whereas $O$. sativa is globally consumed, O. glaberrima is peculiar to the West Africa sub-region (Sweeney and McCouch 2007) and is characterized by specific qualitative and quantitative traits (Gayin and others 2015a), such as a locally preferred taste, excellent weed competitiveness, and the ability to grow in a wide range of difficult ecosystems (Agnoun and others 2012). For these reasons, O. glaberrima is adopted by many African farmers, regardless of its low yields andmarket value, of the susceptibility to shattering, and of poor resistance to lodging (Gayin and others 2015b; Manful and Graham-Acquaah 2016).

In the 1990s the Africa Rice Center (AfricaRice), a leading pan-African rice research organization, developed through conventional cross-breeding between $O$. sativa (upland lines) and O. glaberrima and distributed to local farmers a group of rice varieties, called NERICA (New Rice for Africa) (Nwanze and others 2006). These new varieties combine the high-tillering ability, early maturity, and adaptability to local agronomical conditions of $O$. glaberrima to the high-yields of $O$. sativa, thus allowing for improvement sub-Saharan African farmers' livelihoods.

As for the molecular and rheological characterization of African-grown rice varieties, a high number of studies have focused on starch, the major component of rice grains, and have highlighted its physical, molecular, and thermal properties (Gayin and others 2015a, b). However, especially for O. glaberrima, more information is still required, not only on starch properties and digestibility, but also on what attains protein structure and their overall organization in the rice grains, that remain almost uncharacterized. These investigations may pave the way to the full exploitation of this indigenous variety, and eventually to meet consumers' preferences by introducing added-value products from locally sourced raw materials on the African market. 
The objective of this study was to address the molecular properties of rice protein fractions by combining molecular-based approaches with starch digestibility measurements and with a study on starch thermal and structural properties. This combined information may contribute to the current understanding of the molecular basis of the potential use of different rice species in specific food products.

\section{Materials and methods}

\section{Materials}

Two varieties of rice belonging to the species $O$. glaberrima (G-766 and G-995), two varieties belonging to the species $O$. sativa (SAHEL 208 and WITA 8), and the interspecific rice NERICA L-19 were provided by the Africa Rice Center (Cotonou, Benin). Images of representative samples of $O$. glaberrima and O. sativa are shown in Figure 1A. When appropriate, samples were ground with a laboratory mill (IKA Universal Mühle M20; Janke \& Kunkel GmbH \& Co KG, IKA Laborteknic, Staufen, Germany), fitted with a water cooling jacket in order to avoid overheating during grinding. Apparent amylose content was measured using the standard iodine colorimetric method ISO 6647-2-2011, using an Auto Analyzer 3 (Seal Analytical, Germany) and well-known standard rice varieties (IR65, IR24, IR64 and IR8) as standards. Proximate analyses (humidity, proteins) were carried out according to AACC standard methods, as detailed elsewhere (Marti and others 2010, 2014; Gayin and others 2015a, b). Scanning electron microscopy images of starch granules from representative samples of $O$. glaberrima and $O$. sativa are shown in Figure 1B, and show a similar morphology of starch granules in the two rice species.

\section{Protein characterization}

The solubility of proteins in rice samples was determined by using buffers of various composition, essentially as described by Marti and others (2014). Proteins were extracted by dispersing $0.5 \mathrm{~g}$ of 
finely ground samples $(\leq 0.25 \mathrm{~mm})$ in $10 \mathrm{~mL}$ of $0.05 \mathrm{M}$ sodium phosphate buffer, $\mathrm{pH} 7.0$, containing $0.1 \mathrm{M} \mathrm{NaCl}$. After stirring at room temperature for $60 \mathrm{~min}$ and removal of insoluble materials by centrifugation $\left(10000 \times g, 20 \mathrm{~min}, 20^{\circ} \mathrm{C}\right)$, the protein content in the supernatant was assessed by a dye-binding method (Bradford 1976). Where indicated, the buffer used for protein extraction also contained $6 \mathrm{M}$ urea or $6 \mathrm{M}$ urea and $10 \mathrm{mM}$ dithiothreitol (DTT). Results are expressed as mg soluble proteins $[\mathrm{g} \text { rice flour }]^{-1}$.

A given amount (typically, $0.015 \mathrm{mg}$, as assessed by the dye-binding protein assay) of the proteins solubilised from rice samples in the presence/absence of urea and DTT (see above) was diluted $(1 / 1 \mathrm{v} / \mathrm{v})$ with SDS-PAGE denaturing buffer $(0.125 \mathrm{M}$ Tris-HCl, $\mathrm{pH} 6.8,50 \%$ glycerol, 1.7\% sodium dodecyl sulphate; $0.01 \%$ Bromophenol Blue) containing 1\% (v/v) 2-mercaptoethanol when indicated, and heated at $100{ }^{\circ} \mathrm{C}$ for $10 \mathrm{~min}$. The electrophoretic runs were carried out in a 12\% monomer fixed porosity gel using a MiniProtean apparatus (Bio-Rad, Richmond, VA, USA). Gels were stained with Coomassie Brilliant Blue (Barbiroli and others 2013).

Accessible thiol groups were determined as in Barbiroli et al. (2015), by suspending $0.5 \mathrm{~g}$ of finely ground rice samples in $10 \mathrm{~mL}$ of $0.05 \mathrm{M}$ sodium phosphate, $0.1 \mathrm{M} \mathrm{NaCl}, \mathrm{pH} 7.0$, containing $0.2 \mathrm{mM} 5,5^{\prime}$-dithiobis-(2-nitrobenzoate) (DTNB, Ellman, 1959), in the presence/absence of $6 \mathrm{M}$ urea. After stirring for $60 \mathrm{~min}$ at $25^{\circ} \mathrm{C}$, the suspension was centrifuged $\left(10000 \times \mathrm{g}, 20 \mathrm{~min}, 20^{\circ} \mathrm{C}\right)$ and the absorbance of the supernatant was read at $412 \mathrm{~nm}$ against a proper blank. Results are expressed as $\mu \mathrm{mol}$ thiols $[\mathrm{g} \text { rice flour }]^{-1}$.

\section{Pasting properties}

Rice pasting properties were measured in a Brabender Micro-ViscoAmyloGraph (Brabender, Duisburg, Germany) on finely ground samples, according to a slight modification of the procedure in Marti et al. (2010). An aliquot of rice flour (12 g) was dispersed in $100 \mathrm{~mL}$ of distilled water, scaling both flour and water weight on a $14 \%$ flour moisture basis. The pasting properties were evaluated under constant instrumental conditions (speed: $250 \mathrm{rpm}$; sensitivity: $300 \mathrm{cmg}_{\mathrm{f}}$ ) by using 
the following time-temperature profile: heating from $30{ }^{\circ} \mathrm{C}$ up to $95{ }^{\circ} \mathrm{C}$; holding at $95{ }^{\circ} \mathrm{C}$ for 20 min; cooling from $95{ }^{\circ} \mathrm{C}$ to $30^{\circ} \mathrm{C}$. Heating and cooling were carried out at a rate of $3{ }^{\circ} \mathrm{C} / \mathrm{min}$. Pasting parameters were calculated by using a specific software (Viscograph, version 2.3.7).

\section{In vitro Starch Digestibility}

The method of Englyst (Englyst and others 2000) was used to assess in vitro carbohydrate digestibility on cooked rice grains by means of the estimation of rapidly (RDS) and slowly (SDS) digestible starch fractions that are likely to become available for rapid or slow absorption from the small intestine, thus modulating glycemic response. Rapidly (RDS) and slowly (SDS) digestible starch fractions were calculated from the glucose released at $20 \mathrm{~min}$ and between 20 and at $120 \mathrm{~min}$, respectively, as determined by HPLC (Marti and others 2017). Hydrolytic enzymes were from Sigma Aldrich (St. Louis, MO, USA): pancreatin from porcine pancreas, EC 232.468.9, Sigma P7545; amyloglucosidase from Aspergillus niger, EC 3.2.1.3, Sigma A7095. Two sets of data from independent cooking trials were averaged. RDS and SDS fractions are expressed as percentage of total available starch (RDS + SDS).

\section{Characterization of starch fragments from enzymatic hydrolysis by SE-HPLC}

Rice flour samples were heated up to the microviscoamylographic gelatinization peak (see above). At this point, the run was stopped and the samples were immediately frozen in liquid nitrogen. After freeze-drying, an aliquot of each sample $(100 \mathrm{mg})$ was dispersed in $3 \mathrm{~mL}$ of $0.05 \mathrm{M}$ sodium acetate buffer, $\mathrm{pH} 6.0$, and incubated for $24 \mathrm{~h}$ at $37^{\circ} \mathrm{C}$ in the absence of enzymes or in the presence of either 10-11 U of pullulanase (EC 3.2.1.41, from Bacillus acidopullulyticus, Sigma P2986) or of 10-11 U of $\alpha$-amylase (EC 3.2.1.1, from Bacillus spp., Sigma A6814). At the end of the incubation period, samples were spun for $10 \mathrm{~min}$ at $10,000 \times g, 20^{\circ} \mathrm{C}$. Supernatants were filtered through a $0.22 \mu \mathrm{m}$ filter, and $0.2 \mathrm{~mL}$ of the filtrate were loaded into a HPLC system (515 pump, Dual Absorbance detector 2487, Waters Co., Milford, MA, USA), connected in series to a differential refractometer 
154

155

156

157

158

159

160

161

162

163

164

165

166

167

168

169

170

171

172

173

174

175

176

177

178

179

(Optilab T-rEX, Wyatt Co., Santa Barbara, CA, USA) and to a Multi Angle Light Scattering (MALS) instrument (DAWN HELEOS, Wyatt Co., Santa Barbara, CA, USA). Polysaccharides were fractionated on a size-exclusion column (Ultrahydrogel ${ }^{\mathrm{TM}}$ Linear $7.8 \times 300 \mathrm{~mm}$, Waters Co., Milford, MA, USA), by using $0.05 \mathrm{M}$ sodium acetate, $\mathrm{pH} 6.0$ as the eluant, at a flow rate of 0.4 mL/min. The ASTRA software (ASTRA V 5.1.9.1, Wyatt Technology Co., Santa Barbara, CA, USA) was used for data analysis.

\section{NMR experiments}

NMR spectra were acquired at room temperature on a Bruker AVANCE-600 spectrometer (Bruker Spectrospin GmbH, Rheinstetten, Germany), operating at $600.1 \mathrm{MHz}$ (proton frequency) and equipped with a $4 \mathrm{~mm}$ broad-band Cross-Polarisation Magic Angle Spinning (CP-MAS) probe for solid state measurements. Flour samples were directly pressed into a $4 \mathrm{~mm} \mathrm{ZrO}_{2}$ rotor without preliminary treatment. Natural abundance ${ }^{13} \mathrm{C}$ spectra were acquired at $150.9 \mathrm{MHz}$ while spinning samples at $10 \mathrm{kHz}$ (Pines and others 1973). Proton decoupling was achieved with a GARP-based composite pulse. Relevant acquisition parameters were: spectral width $45.4 \mathrm{kHz}$; acquisition time $11 \mathrm{~ms}$; relaxation delay 2s (fast acquisition conditions); contact time for Cross Polarization $1.5 \mathrm{~ms}$; number of scans 3600 . Adamantane was used as external ${ }^{13} \mathrm{C}$ chemical shift reference, by setting the resonance of the most intense band at $38.56 \mathrm{ppm}$.

\section{DSC measurements}

Differential Scanning Calorimetry (DSC) measurements were carried out in the $20-150^{\circ} \mathrm{C}$ range at a scanning rate of $2.0^{\circ} \mathrm{C} / \mathrm{min}$ in a Perkin-Elmer DSC6 calorimeter (Waltham, Massachusetts, USA). Indium was used for calibration and distilled water as reference. An aliquot of flour ( $5 \mathrm{~g}$ ) was added to distilled water to give $73 \%$ moisture and thoroughly manually mixed. A $30 \mathrm{mg}$ aliquot of the dough mass was placed in a $0.06 \mathrm{~mL}$ measuring cell. Raw calorimetric data were analyzed with the dedicated software IFESTOS (Fessas and Schiraldi 2000). Two heating-cooling cycles 
were applied to each sample. The average trend of the DSC record of the immediate re-heating run was used as the base-line for elaboration of each given DSC trace. The instrument output signal was converted into apparent specific heat and was scaled with respect to the baseline to obtain the trend of the excess (with respect the pre-gelatinization state) specific heat trace, $C p^{e x}(T)\left[\mathrm{J} \cdot \mathrm{K}^{-1} \cdot \mathrm{g}^{-1}\right]^{-1}$ of the sample (per gram of dry matter), which in turn allowed evaluation of the enthalpy drop $\Delta H$ by a straightforward integration of the corresponding trace (Fessas and others 2008). Gelatinization onset was calculated as the flex point tangent interception with the temperature in the gelatinization peak. Errors were evaluated on at least three replicates.

\section{Statistical analysis}

All tests and measurements were carried out at least in triplicate. Analysis of variance (ANOVA) was performed with Statgraphics XV version 15.1.02 (StatPoint Inc., Warrenton, VA, USA). Samples were used as factor. When the factor effect was significant $(\mathrm{p} \leq 0.05)$, differences among the respective means were determined using Fisher's Least Significant Difference (LSD) test.

\section{Results and Discussion}

\section{Pasting properties}

Pasting properties provide information on starch properties and mutual arrangement of starch components during gelatinization and retrogradation and might allow prediction of the starch behaviour during processing and its suitability for food-related applications (Marti and others 2011). The pasting profiles of the various rice varieties are presented in Figure 2, and values of the most representative properties are reported in Table 1. Samples from O. sativa varieties SAHEL 208 and WITA 8 gave viscoamylographic tracings with a high viscosity peak $(774 \pm 27$ and $723 \pm$ 16 Brabender Units (BU) respectively), suggesting the presence of starch granules with a high swelling capacity, as previously observed in other rice flours (Marti and others 2010). 
In spite of a similar amylose content and of similar morphological traits, a specific viscosity peak is either almost absent (G-995) or very low (G-766) in the case of O. glaberrima. In the absence of microstructural differences among starch granules in the two species (Fig. 1B), this may relate to the properties of amylopectin chains (Park and others 2007; Vandeputte and others 2003). In the case of NERICA, the viscosity peak $(788 \pm 21 \mathrm{BU})$ appears similar to that of $O$. sativa, but viscosity dramatically decreases immediately after the gelatinization peak to values similar to those of O. glaberrima, suggesting a lower ability of starch in NERICA to withstand heating and shear stress during cooking.

Species-specific differences were assessed during the holding period at $95{ }^{\circ} \mathrm{C}$, when high temperatures and mechanical shear may lead to starch granule disruption and amylose leaching. Indeed, during the holding time, viscosity decreases with a similar tendency in the case of $O$. sativa samples, whereas it appears stable in the case of the two O. glaberrima varieties. Although viscosity increases for both cultivars because of retrogradation, this increase is more evident in G-766. This may result from the different starch gelatinization level detected during the heating step, that in turn might be related to a cultivar-dependent organization within the starch granules, as pointed out in recent studies on various $O$. glaberrima accessions (Gayin and others 2015) and in studies that compared cultivars not considered here (Gayin and others $2016 \mathrm{a}, \mathrm{b}$ ).

\section{Organization of the protein network}

Protein aggregation studies provide information on protein structural features and on the nature of inter-protein interactions in cereal-based materials (Moroni and others 2010). In particular, the role of hydrophobic interactions and of disulfide bonds in the stabilization of protein aggregates can be addressed by extracting proteins in the absence/presence of denaturants and of disulfide-breaking agents (Marengo and others 2015; Barbiroli and others 2013) in various media. Results from $O$. sativa (SAHEL 208, and WITA 8), O. glaberrima (G-766, and G-995), and NERICA L-19 are shown in Figure 3A. 
The amount of soluble proteins appears comparable in all samples in the presence/absence of urea, whereas the presence of dithiothreitol (DTT, a disulfide-breaking agent) increases the amount of proteins solubilized from $O$. glaberrima much more than in other samples. This highlights a major role of inter-protein disulfide bonds in stabilizing a protein network in $O$. glaberrima. The effect of DTT on the amount of proteins extracted from $O$. sativa and NERICA was comparable, suggesting that this feature is controlled by $O$. sativa genetic traits in the NERICA hybrid. Given the modest differences in the overall protein pattern between $O$. glaberrima and $O$. sativa (see below), it is possible that these traits govern the rate of synthesis or deposition of individual components. Different rates of synthesis for specific proteins may affect intermolecular interactions, as observed in wheat (Iametti and others, 2006; 2013).

Protein attitude to network formation - relevant to technological behaviour - is affected by the amount and accessibility of protein thiols that can undergo disulphide exchange reactions when with existing protein disulfides (Barbiroli and others 2013). The amount of accessible thiols in the different rice samples as a function of presence/absence of urea is shown in Figure 3B. Since the total cysteine contents in $O$. glaberrima and $O$. sativa is comparable (3.37 and 3.13 percent of total aminoacids, respectively, equivalent to 0.31 and $0.26 \mathrm{~g}$ cysteine in $100 \mathrm{~g}$ of the original sample), the observed marked differences should relate to structural features of the proteins in each species. The number of accessible thiol groups in the presence/absence of denaturant is highest in $O$. glaberrima samples. Cysteine thiols in O. glaberrima are more exposed - regardless of the presence of chaotropes - and participate to a higher number of interprotein disulfide bonds (as indicated by the conditional solubility studies reported in Figure 3A) than in O. sativa. The amount of thiols detected in NERICA L-19 in the presence and absence of chaotropes confirms that protein aggregation in this hybrid strongly depends on $O$. sativa traits.

To assess the nature of proteins present in the various samples and to verify whether some specific proteins were preferentially involved in aggregation events, proteins solubilized in the various media were separated by SDS-PAGE. As shown in Figure 4, differences between rice 
species under investigation were mostly limited to the relative abundance of individual components within a given protein family. Thus, the same polypeptides contribute to the protein pattern of the different rice species. However, the conditional solubility and thiol accessibility studies reported above suggest that these proteins may acquire a species-specific folding (as indicated by thiol accessibility data), and may interact among themselves with species-specific bonds (as indicated by the solubility data in Fig. 3A). Hydrophobic bonds play a major role in stabilizing buffer-insoluble aggregates in $O$. sativa and NERICA, whereas disulfide bonds are preferentially responsible for aggregate formation and/or stability in O. glaberrima.

\section{Structural features of starch: DSC and NMR measurements}

Since pasting properties and protein overall structural studies highlighted species-specific differences, one sample for each species (namely, WITA 8, G-766, and NERICA L-19) underwent further investigation of details of the starch structure and behaviour by using both DSC and solidstate ${ }^{13} \mathrm{C} C P / M A S ~ N M R$. These approaches have been widely used to characterize starches in native food products (Bertocchi and Paci 2008; Cheetham and Tao 1998; Tester and others 1998).

As shown in Figure 5, all the DSC profiles present signals ensuing from starch gelatinization (at low temperature) and from amylose-lipid dissociation (at high temperature). Both these events are strongly dependent on water availability (Schiraldi and Fessas, 2003). Here, the presence of excess water and the slow scanning rate allowed good discrimination of the observable transitions.

Although the overall enthalpies were similar $(14.3 \pm 0.7,15.6 \pm 0.7,13.7 \pm 0.7 \mathrm{~J} / \mathrm{g}$, for WITA 8, G-766, and NERICA L-19, respectively), the gelatinization profile for $O$. sativa was markedly sharper than that observed for the other two samples (half-height width of $4.5,6.0,8.0{ }^{\circ} \mathrm{C}$, for O. sativa, NERICA L-19, and O. glaberrima, respectively). This may be taken as an indication of a more homogenous distribution of smaller-sized starch granules in O. sativa, in accordance with results from enzymatic hydrolysis activities (see below). 
The gelatinization onset temperatures $\left(72.8 \pm 0.5,73.1 \pm 0.5,75.5 \pm 0.5{ }^{\circ} \mathrm{C}\right.$, for $O$. sativa, NERICA L-19, and O. glaberrima, respectively) essentially confirm recent and detailed reports (Gayin and others 2016a, 2016b). Some of these recent studies also provided evidence of a difference in the macromolecular organization within $O$. glaberrima starch granules, that had higher ratio of absorbance to scattering than $O$. sativa when exposed to iodine vapor. This result was interpreted in terms of greater flexibility and availability of glucan chains to form complexes with iodine in $O$. glaberrima as compared to $O$. sativa (Gayin and others 2016b). Finally, the amyloselipid dissociation process seems very similar in all samples.

On these basis, it seemed worth to explore whether the subtle differences reported in previous studies could be interpreted and/or explained in improved detail by using solid-state ${ }^{13} \mathrm{C}$ NMR. Figure 6 shows an expansion (in the $C_{1}$ carbon region) of the CP-MAS ${ }^{13} \mathrm{C}-\mathrm{NMR}$ spectra taken at room temperature on untreated rice flours obtained from some of the same cultivars of $O$. glaberrima, O. sativa, and NERICA used for DSC studies. Resonances corresponding to the various individual polymorphs appear to be present in varying amounts. Both A- and B-type starch are distinguishable on the basis of their characteristic resonance patterns (respectively, a triplet and a doublet of resonances with even intensities). Such features arise from the different number of nonequivalent glucose monomers within the crystalline cell unit. Residues at the interface of the helical regions of both A- and B-type polymorphs also give another well resolved resonance at $102.4 \mathrm{ppm}$, accompanied by a broad signal at about 98 ppm (Paris and others 1999). The structural differences made evident by room-temperature CP-MAS ${ }^{13} \mathrm{C}-\mathrm{NMR}$ studies may affect the behaviour of starch when exposed to higher temperatures in the presence of enough water to allow starch gelatinization. Detailed studies on the kinetics and equilibria of these events are currently underway.

Accessibility of gelatinized starch to hydrolytic enzymes and characterization of fragments from enzymatic starch hydrolysis 
The susceptibility of starch to specific enzymatic attack can provide information on structural differences among samples (Miao and others 2011), that may depend on starch granule size, composition (i.e., the amylose/amylopectin ratio), and on physical architecture and porosity (Lehmann and Robin, 2007; Naguleswaran and others 2014). Accessibility to amylase action in the cooked whole grains, as estimated by the Englyst's method, allows to distinguish between readily digestible (RDS), slowly digestible (SDS), and resistant starch (RS). These parameters may be used to estimate the potential glycemic response of foods (Englyst et al 1999; EFSA 2011). Glycemic response appear to be directly related to RDS, whereas insulin demand was shown to be inversely correlated to SDS (Garsetti et al, 2002).

In spite of the microstructural similarity between starch granules in the two species (Fig. 1B), O. glaberrima had a much lower RDS content than $O$. sativa $(69.8 \pm 2.8$ vs $80.9 \pm 2.6 \mathrm{~g} / 100 \mathrm{~g}$ available starch). NERICA showed an intermediate RDS content $(75.7 \pm 4.3 \mathrm{~g} / 100 \mathrm{~g}$ available starch). Thus, O. glaberrima has a much lower predicted glycaemic index than $O$. sativa, in accordance with other very recent reports (Gayin and others 2017),

In a different approach, aiming at assessing the behaviour of starch in rice flours used as ingredient of other food products, suspensions of rice flours were heated until gelatinization, as assessed by microviscoamylographic tests (see Fig. 2). The gelatinized samples were then treated overnight with $\alpha$-amylase or with pullulanase, a debranching enzyme that cleaves $\alpha-1,6$ linkages in amylopectin molecules (Lin and Chang 2006). Effects of the various enzymatic treatments were then studied by analysing the chromatographic pattern of soluble polysaccharides.

The size distribution of soluble molecules released by action of the two enzymes on the gelatinized products was addressed by SE-HPLC in combination with Static (Multi Angle) Light Scattering, and compared to the pattern of soluble polysaccharides in samples incubated in the same condition, but in the absence of enzymes. This approach is useful to highlight differences in the hydrolysis pattern of the rice samples that may result from a different structural organization of starch components in the various rice species (Barbiroli and others 2013). Indeed, differences in the 
distribution and length of amylopectin lateral chain length may lead to different hydrolysis patterns with pullulanase, that is reportedly more effective on short amylopectin branches (Liu and others 2015).

As shown in the top panel in Figure 7, the SE-HPLC pattern of soluble polysaccharides present in the gelatinized materials in the absence of enzymatic treatments is different. Although the response from the refractive index detector used to analyse the chromatographic profile is not strictly quantitative, there is some indication that only a modest amount of large-sized soluble fragments is present in the gelatinized starch from $O$. glaberrima, confirming indications from cooking losses studies (Gayin and others 2017). Sensibly smaller fragments - present in larger amounts - formed the majority of the soluble material solubilized from gelatinized $O$. sativa samples, whereas NERICA gave a chromatographic profile intermediate between that of $O$. sativa and of $O$. glaberrima.

The action of $\alpha$-amylase on gelatinized samples resulted in the formation of soluble polymers with a very similar size distribution (centered around 4000-5000 Da, equivalent to about 25-30 glucose units). With the limitations discussed before, the intensity of the peak in the $O$. glaberrima tracings in the middle panel of Figure 7 is lower than in O. sativa and in NERICA, supporting previous observations on the lower sensitivity of $O$. glaberrima starch to $\alpha$-amylase as a consequence of its crystallinity (Gayin and others 2017).

As shown in the bottom panel of Figure 7, pullulanase action on the various gelatinized samples gave two major peaks - at 23 and 25 minutes - for all samples, indicative of the release of small-sized oligosaccharides in addition to larger species (Barbiroli and others 2013). However, the amount of these peculiar fragments appears species-specific, and the highest amount of the smallest released molecules was detected in the case of $O$. glaberrima. This is consistent with the reported presence of a higher level of short B-chains of the so-called fingerprint-type in O. glaberrima with respect to O. sativa and NERICA (Gayin and others 2016a). In all cases, the release of soluble relatively small-sized glucose oligomers appears to stem from the action of pullulanase on 
otherwise insoluble amylopectin components, and offers circumstantial support for external amylopectin chains being longer in $O$. glaberrima than in the other two species, as suggested by Gayin and others (2016a).

Indeed, a comparison between the tracings in the various panels of Figure 7 also makes it clear that both pullulanase and amylase are capable to break down the large soluble polysaccharides that are already present in the gelatinized materials before any enzymatic treatment. This breakdown is almost complete in the case of amylase, as discussed above. Residual soluble fragments of apparent size around $10^{5} \mathrm{Da}$ are still present in all cases after treatment with pullulanase, but these large species are much more evident after treatment of gelatinized samples from $O$. sativa and NERICA.

\section{Conclusions}

The approaches presented here provide useful insights as for the structural features of proteins and starch in rice species grown in Africa. The overall protein structural organization in $O$. glaberrima appears much different from the one in O. sativa. Proteins in O. glaberrima are organized in polymeric forms mainly stabilized by inter-protein disulphide bonds, at contrast with hydrophbobic interactions being the dominating type of interaction in protein aggregates in both O. sativa and NERICA, where these interaction impairs thiol accessibility much more than in O. glaberrima. Differences in molecular starch structure among the various species are reflected in their starch gelatinization behaviour. Structural features of the gelatinized starch in the various species indicate that soluble fractions were least present in O. glaberrima, in particular when considering the amylopectin component. This provides additional molecular-based rationale for the reportedly low potential glycaemic index of $O$. glaberrima. All together, the properties of starch and proteins in the NERICA variety appear very similar to those of $O$. sativa. The results presented here may provide some useful guidelines for extending the use of $O$. glaberrima cultivars (and of their crosses) in the 
design and production of new types of rice-based products, that could expand the market for locally sourced materials in Africa.

\section{Acknowledgements}

This work was supported by the "New Products" Project, in the frame of the Global Rice Science Partnership (GRiSP).

\section{Authors' Contributions}

Authors M. Marengo, A. Barbiroli, J. A. Hogenboom, and S. Iametti conducted the experiments dealing with protein characterization. Authors M. C. Casiraghi, A. Marti, M. A. Pagani, and A. Barbiroli conducted the experiments related to starch characterization. Authors J. Manful, S. Graham-Acquaah, F. Bonomi, and S. Iametti designed the experiments. Authors E. Ragg and D. Fessas conducted the NMR and DSC experiments, respectively. Authors S. Iametti, A. Marti, J. Manful and F. Bonomi interpreted the results and wrote the manuscript.

\section{References}

Agnoun Y, Biaou SSH, Sié M, Vodouhè RS, Ahanchédé A. 2012. The African Rice Oryza glaberrima Steud: Knowledge, distribution and prospects. Int J Biol 4(3):158-80.

Barbiroli A, Bonomi F, Casiraghi MC, Iametti S, Pagani MA, Marti A. 2013. Process conditions affect starch structure and its interactions with proteins in rice pasta. Carbohydr Polym 92(2):1865-72.

Bertocchi F, Paci M. 2008. Applications of high-resolution solid-state NMR spectroscopy in food science. J Agric Food Chem 56(20):9317-27.

Bradford MM. 1976. A rapid and sensitive method for the quantitation of microgram quantities of protein utilizing the principle of protein dye-binding. Anal Biochem 72(1):248-54. 
Cabrera-Chavez F, Calderon de la Barca AM, Islas-Rubio AR, Marti A, Marengo M, Pagani MA, Bonomi F, Iametti S. 2012. Molecular rearrangements in extrusion processes for the production of amaranth-enriched, gluten-free rice pasta. LWT-Food Sci Technol 47(2):421-6.

Cheetham NWH, Tao L. 1998. Solid state NMR studies on the structural and conformational properties of natural maize starches. Carbohydr Polym 26 (4):285-92.

EFSA. 2011. Scientific Opinion on the substantiation of a health claim related to "slowly digestible starch in starch-containing foods" and "reduction of post-prandial glycaemic responses" pursuant to Article 13(5) of Regulation (EC) No 1924/2006.1. EFSA Journal 9:2292.

Ellman GL. 1959. Tissue sulfhydryl groups. Arch Biochem Biophys 82(1):70-7.

Englyst KN, Englyst HN, Hudson GJ, Cole TJ, Cummings JH. 1999. Rapidly available glucose in foods: an in vitro measurement that reflects the glycemic response. Am J Clin Nutr 69(3):44854.

Englyst KN, Hudson GJ, Englyst HN. 2000. Starch analysis in food. Encyclopedia of Analytical Chemistry. RA Meyers, editor. Chichester, UK: John Wiley and Sons Ltd. p. 4246-4262.

Fessas D, Schiraldi A. 2000. Starch gelatinization kinetics in bread dough. DSC investigations on simulated baking processes. J Therm Anal Calorim 61(2):411-23.

Fessas D, Signorelli M, Pagani MA, Mariotti M, Iametti S, Schiraldi A. 2008. Guidelines for buckwheat enriched bread. Thermal analysis approach. J Therm Anal Calorim 91(1):9-16.

Garsetti M, Vinoy S, Lang V, Holt S, Loyer S, Brand-Miller JC. 2005. The glycemic and insulinemic index of plain sweet biscuits: relationships to in vitro starch digestibility. J Am Coll Nutr 24(6):441-7.

Gayin J, Abdel-Aal EM, Manful J, Bertoft E. 2016a. Unit and internal chain profile of African rice (Oryza glaberrima) amylopectin. Carbohydr Polym 137:466-472.

Gayin J, Bertoft E, Manful J, Yada RY, Abdel-Aal EM. 2016b. Molecular and thermal characterization of starches isolated from African rice (Oryza glaberrima). Starch/Stärke, 68(12):9-19. 
Gayin J, Chandi GK, Manful J, Seetharaman K. 2015a. Classification of rice based on statistical analysis of pasting properties and apparent amylose content: The case of Oryza glaberrima accessions from Africa. Cereal Chem 92(1):22-8.

Gayin J, El-Sayed M, Abdel-Aal EM, Manful J, Bertoft E, Marcone M, Ragaee S. 2017. Physical, cooking and thermal properties of African rice (Oryza glaberrima) and its starch digestibility in vitro. LWT - Food Sci Technol 75:481-7.

Iametti S, Bonomi F, Pagani MA, Zardi M, Cecchini C, D’Egidio MG. 2006. Properties of the protein and carbohydrate fractions in immature wheat kernels. J Agric Food Chem 54:10239-44.

Iametti S, Marengo M, Miriani M, Pagani MA, Marti A, Bonomi F. 2013. Integrating the information from proteomic approaches: A "thiolomics" approach to assess the role of thiols in protein-based networks. Food Res Int 54: 980-7.

Lehmann U, Robin F. 2007. Slowly digestible starch - its structure and health implications: a review. Trends Food Sci Technol 18(7):346-55.

Lin JH, Chang YH. 2006. Effects of type and concentration of polyols on the molecular structure of corn starch kneaded with pullulanase in a Farinograph. Food Hydrocoll 20(2-3):340-7.

Liu G, Hong Y, Gu Z, Li Z, Cheng L. 2015. Pullulanase hydrolysis behaviors and hydrogel properties of debranched starches from different sources. Food Hydrocoll 45:351-60.

Manful JT, Graham-Acquaah S. 2016. African rice (Oryza glaberrima): a brief history and its growing importance in current rice breeding efforts. Encyclopedia of Food Grains ( $2^{\text {nd }}$ edition), C Wrigley, H Corke, K Seetharaman, J Faubion, editors. Oxford, UK: Academic Press. p. 140146.

Marengo M, Bonomi F, Marti A, Pagani MA, Elkhalifa AEO, Iametti S. 2015. Molecular features of fermented and sprouted sorghum flours relate to their suitability as components of enriched gluten-free pasta. LWT-Food Sci Technol 63(1):511-8. 
Marti A, Abbasi Parizad P, Marengo M, Erba D, Pagani MA, Casiraghi MC. 2017. In vitro starch digestibility of commercial gluten-free pasta: the role of ingredients and origin. J Food Sci doi:10.1111/1750-3481.13673

Marti A, Barbiroli A, Marengo M, Fongaro L, Iametti S, Pagani MA. 2014. Structuring and texturing gluten-free pasta: egg albumen or whey proteins? Eur Food Res Technol 238(2):21724.

Marti A, Fongaro L, Rossi M, Lucisano M, Pagani MA. 2011. Quality characteristics of dried pasta enriched with buckwheat flour. Int J Food Sci Technol 46(11):2393-400.

Marti A, Seetharaman K, Pagani MA. 2010. Rice-based pasta: A comparison between conventional pasta-making and extrusion-cooking. J Cereal Sci 52(3):404-9.

Miao M, Zhang T, Mu W, Jiang B. 2011. Structural characterizations of waxy maize starch residue following in vitro pancreatin and amyloglucosidase synergistic hydrolysis. Food Hydrocoll 25(2):214-20.

Moroni AV, Iametti S, Bonomi F, Arendt EK, Dal Bello F. 2010. Solubility of proteins from nongluten cereals: A comparative study on combinations of solubilising agents. Food Chem 121(4):1225-30.

Naguleswaran S, Vasanthan T, Hoover R, Bressler D. 2014. Amylolysis of amylopectin and amylose isolated from wheat, triticale, corn and barley starches. Food Hydrocoll 35:686-93.

Nwanze KF, Mohapatra S, Kormawa P, Keya S, Bruce-Oliver S. 2006. Rice development in subSaharan Africa. J Sci Food Agric 86(5):675-7.

Paris M, Bizot H, Emery J, Buzare' JY, Buléon, A. 1999. Crystallinity and structuring role of water in native and recrystallized starches by ${ }^{13} \mathrm{C}$ CP-MAS NMR spectroscopy. 1: Spectral decomposition. Carbohydr Polym 39(4):327-39.

Park IM, Ibáñez AM, Zhong F, Shoemaker CF. 2007. Gelatinization and pasting properties of waxy and non-waxy rice starches. Starch/Stärke 59(8):388-96. 
484

485

486

487

488

489

490

491

492

493

494

495

496

Pines A, Gibby MG, Waugh JS. 1973. Proton-enhanced NMR of dilute spins in solids. J Chem Phys 59(2):569-90.

Singh A, Singh B, Panda K, Rai VP, Singh AK, Singh SP, Chouhan SK, Rai V, Singh PK, Singh NK. 2013. Wild rices of Eastern Indo-Gangetic plains of India constitute two sub-populations harbouring rich genetic diversity. Plant Omics 6(2):121-7.

Sweeney KM, McCouch S. 2007. New insights into the history of rice domestication. Trends Genet 23(11):579-87.

Tester RF, Debon SJJ, Karkalas J. 1998. Annealing of wheat starch. J Cereal Sci 28(3):259-72.

Vandeputte GE, Derycke V, Geeroms J, Delcour JA. 2003. Rice starches. II. Structural aspects provide insight into swelling and pasting properties. J Cereal Sci 38(1):53-9.

Wani AA, Singh P, Shah MA, Schweiggert-Weisz U, Gul K, Wani, IA. 2012. Rice starch diversity: effects on structural, morphological, thermal, and physicochemical properties - A review. Comp Rev Food Sci Food Safety 11(5):417-36. 
Table 1

498

Features of starch in the various rice samples

499

\begin{tabular}{ccccccc}
\hline \multirow{2}{*}{$\begin{array}{c}\text { Sample } \\
\text { Amylose }\end{array}$} & Pasting & Max viscosity & Final & Breakdown & Setback \\
& total starch & $\left({ }^{\circ} \mathrm{C}\right)$ & $(\mathrm{BU})$ & $(\mathrm{BU})$ & $(\mathrm{BU})$ & $(\mathrm{BU})$ \\
\hline SAHEL 208 & $28.4 \pm 0.9^{\mathrm{b}}$ & $76.6 \pm 0.1^{\mathrm{b}}$ & $774 \pm 27^{\mathrm{d}}$ & $1029 \pm 25^{\mathrm{d}}$ & $373^{\mathrm{c}}$ & $627^{\mathrm{c}}$ \\
WITA 8 & $27.7 \pm 1.0^{\mathrm{ab}}$ & $75.1 \pm 0.3^{\mathrm{a}}$ & $723 \pm 16^{\mathrm{c}}$ & $1050 \pm 14^{\mathrm{d}}$ & $365^{\mathrm{c}}$ & $661^{\mathrm{d}}$ \\
NERICA L-19 & $28.7 \pm 0.9^{\mathrm{b}}$ & $77.3 \pm 0.3^{\mathrm{c}}$ & $788 \pm 21^{\mathrm{d}}$ & $715 \pm 31^{\mathrm{b}}$ & $476^{\mathrm{d}}$ & $433^{\mathrm{b}}$ \\
G-766 & $26.2 \pm 0.7^{\mathrm{a}}$ & $80.6 \pm 0.6^{\mathrm{d}}$ & $331 \pm 29^{\mathrm{b}}$ & $966 \pm 23^{\mathrm{c}}$ & $9^{\mathrm{b}}$ & $641^{\mathrm{cd}}$ \\
G-995 & $26.2 \pm 0.4^{\mathrm{a}}$ & $85.9 \pm 0.8^{\mathrm{e}}$ & $199 \pm 18^{\mathrm{a}}$ & $565 \pm 11^{\mathrm{a}}$ & $0^{\mathrm{a}}$ & $356^{\mathrm{a}}$
\end{tabular}

$500 \quad$ Values in the same column with the same letters are not significantly different (LSD; $\leq \leq 0.05)$

501 


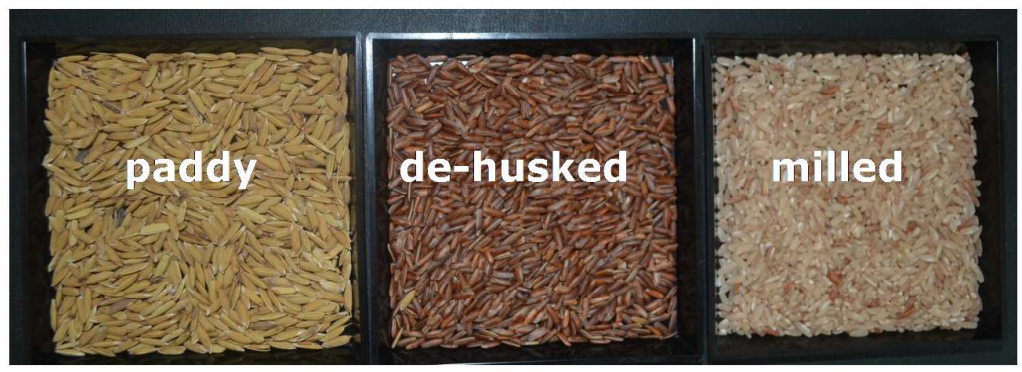

O. glaberrima

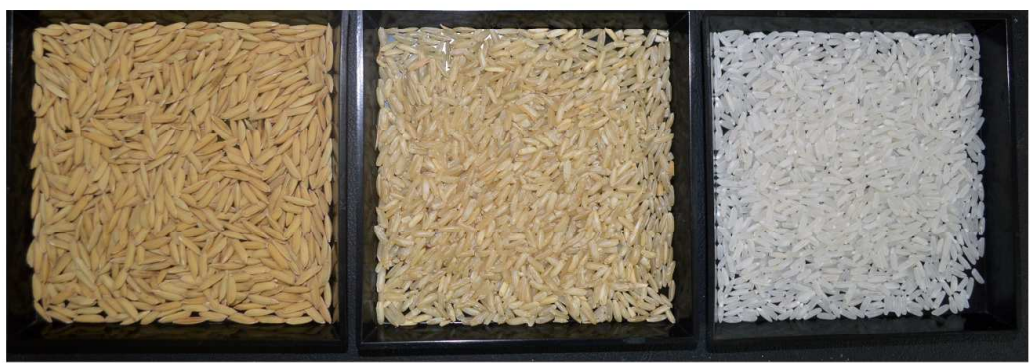

O. sativa

511

512

513

514
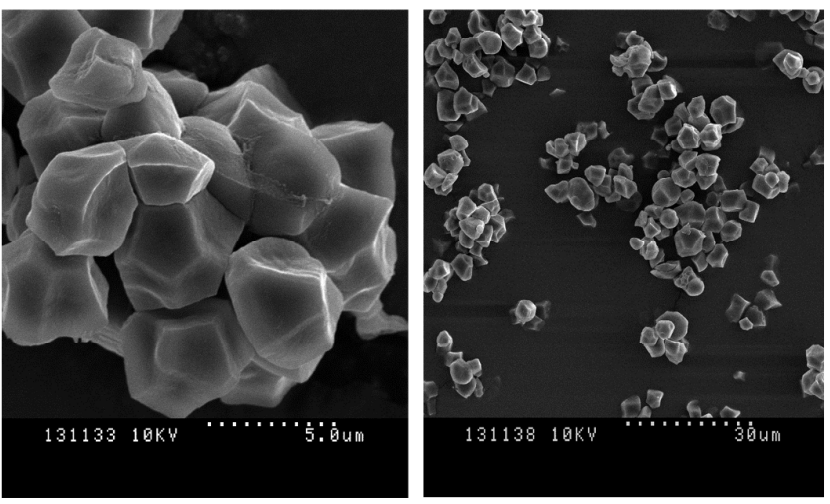

O. glaberrima
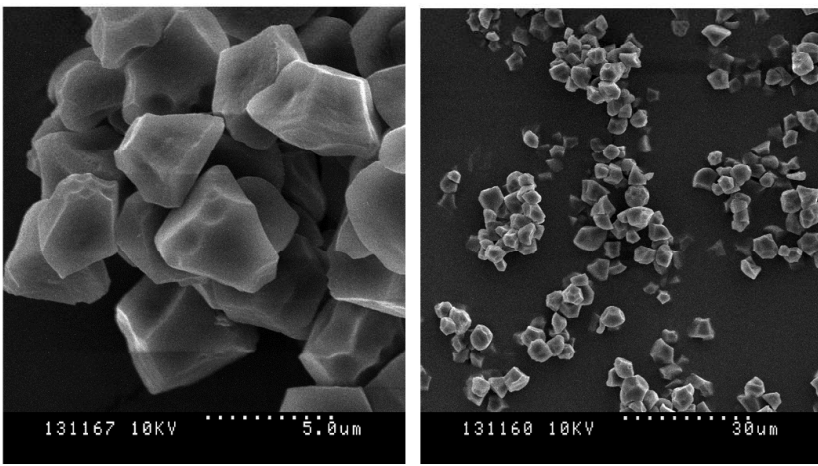

O. sativa

525 Figure 1 - A: morphology of $O$. glaberrima and $O$. sativa grains. B: Scanning electron microscopy

526 images of starch granules from O. glaberrima and O. sativa. 


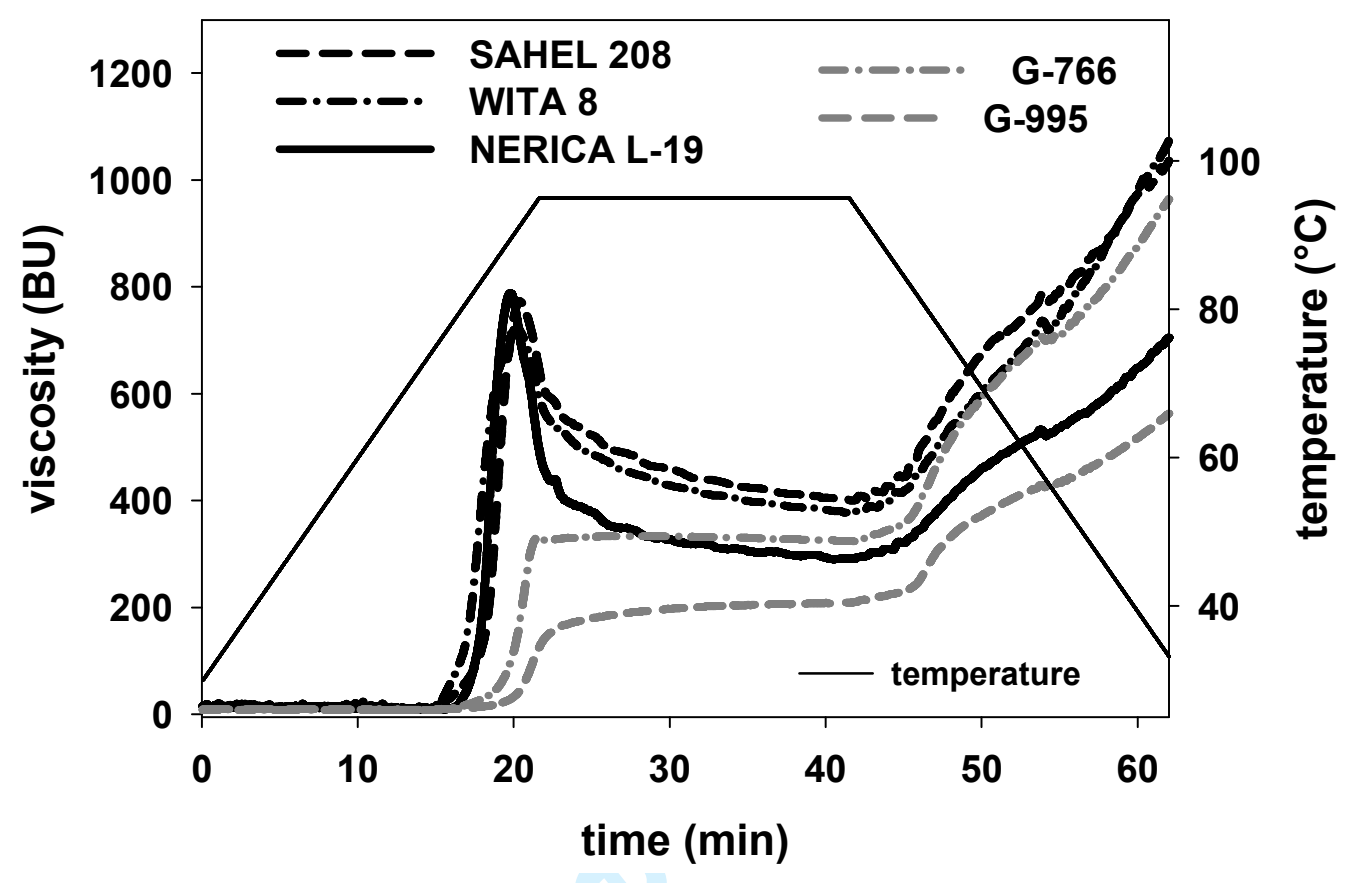

531 Figure 2 - Pasting properties of rice samples. The thin black line is the temperature profile. The

532 thick lines are the pasting profiles: grey, O. glaberrima; solid black line, NERICA; other black 533 lines, O. sativa. 

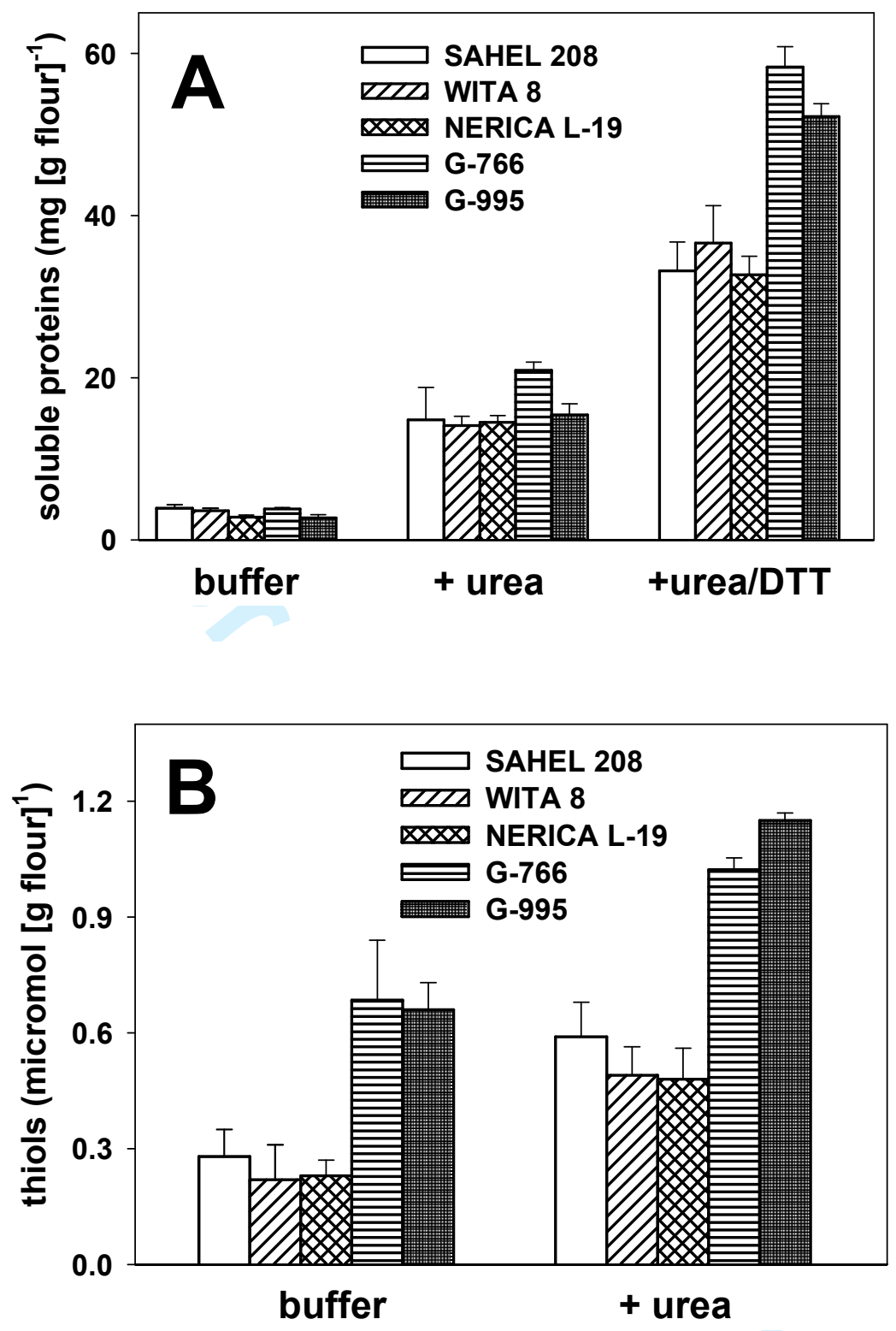

Figure 3 - A: Solubility of proteins from rice in different media. Aliquots of the various samples were suspended under stirring in $0.05 \mathrm{M}$ sodium phosphate, $0.1 \mathrm{M} \mathrm{NaCl}, \mathrm{pH} 7.0$, in the presence/absence of $6 \mathrm{M}$ urea and of $10 \mathrm{mM} \mathrm{DTT}$, as indicated. B: Accessibility of protein thiols in the various rice samples. Thiols were assessed on rice flour samples suspended in $0.05 \mathrm{M}$ sodium phosphate, $0.1 \mathrm{M} \mathrm{NaCl}, \mathrm{pH} 7.0$, in the presence/absence of $6 \mathrm{M}$ urea as indicated. 

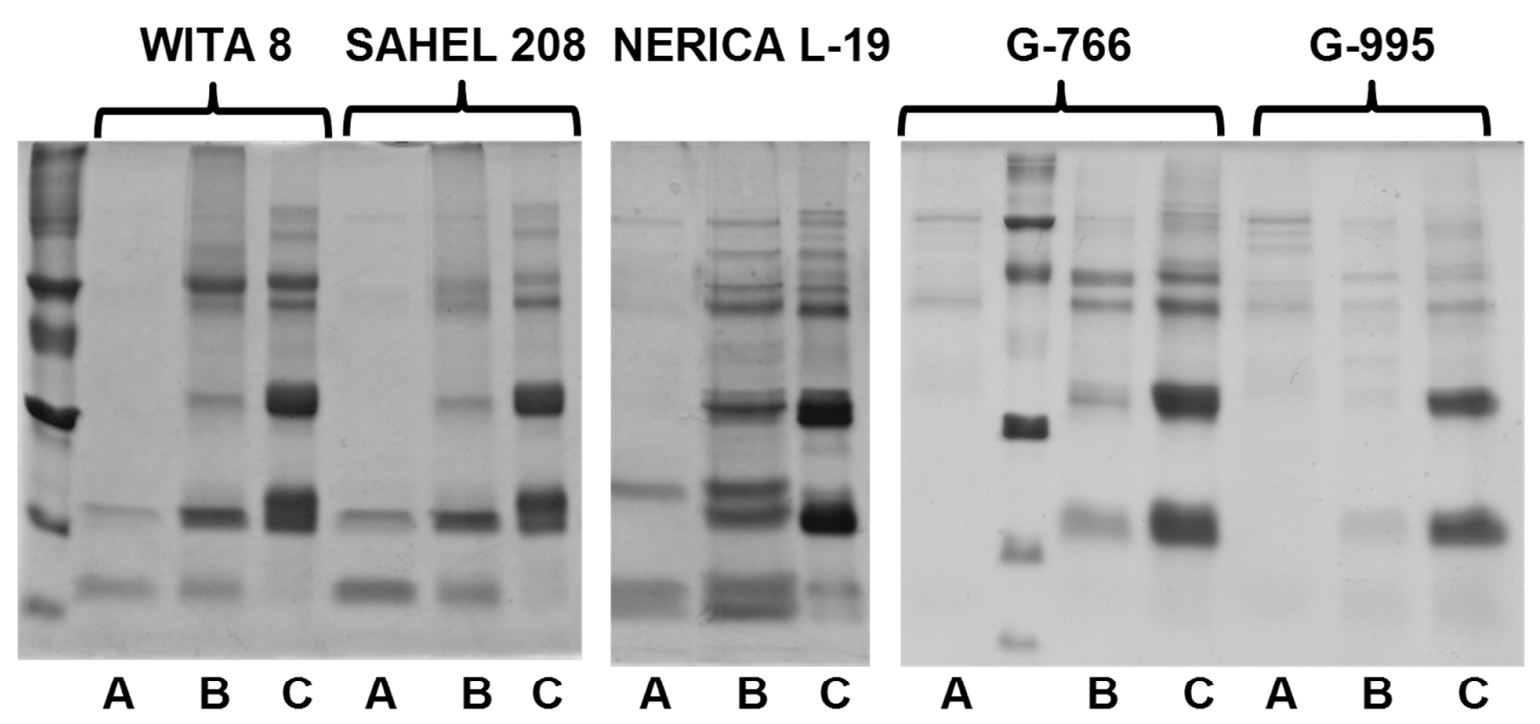

545

546

547 Figure 4 - SDS-PAGE of proteins solubilized in different media. Proteins were extracted by using

$548 \quad 0.05 \mathrm{M}$ sodium phosphate, $0.1 \mathrm{M} \mathrm{NaCl}, \mathrm{pH} 7.0(\mathrm{~A})$, to which $6 \mathrm{M}$ urea (B), or of $6 \mathrm{M}$ urea and 10 549 mM DTT (C) were added. 


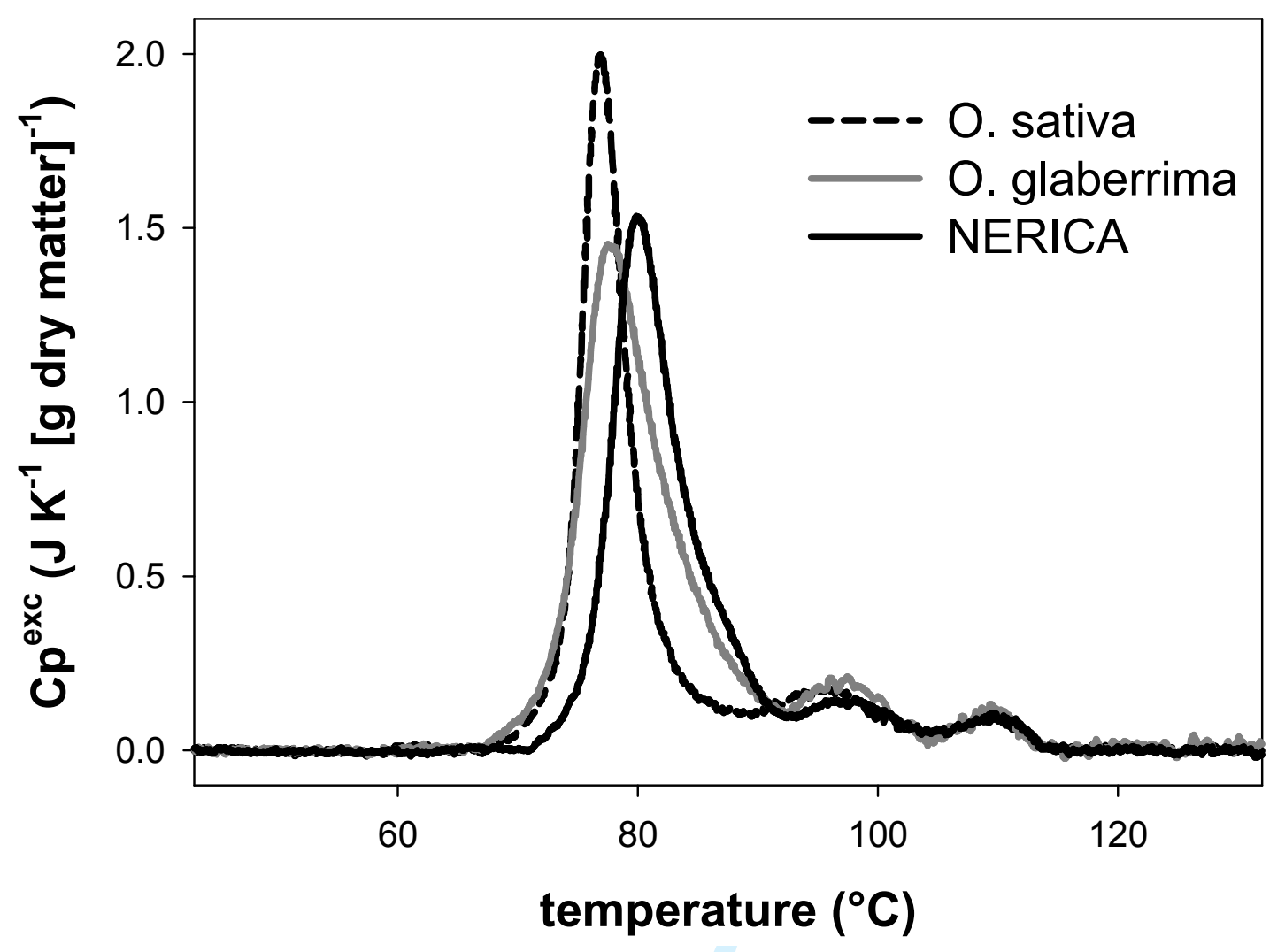

551

552

553 Figure 5 - DSC tracings for the various rice flours $\left(73 \%\right.$ moisture, scan rate $\left.2{ }^{\circ} \mathrm{C} / \mathrm{min}\right)$. Grey, $O$. 554 glaberrima; dashed black, O. sativa; solid black, NERICA. 


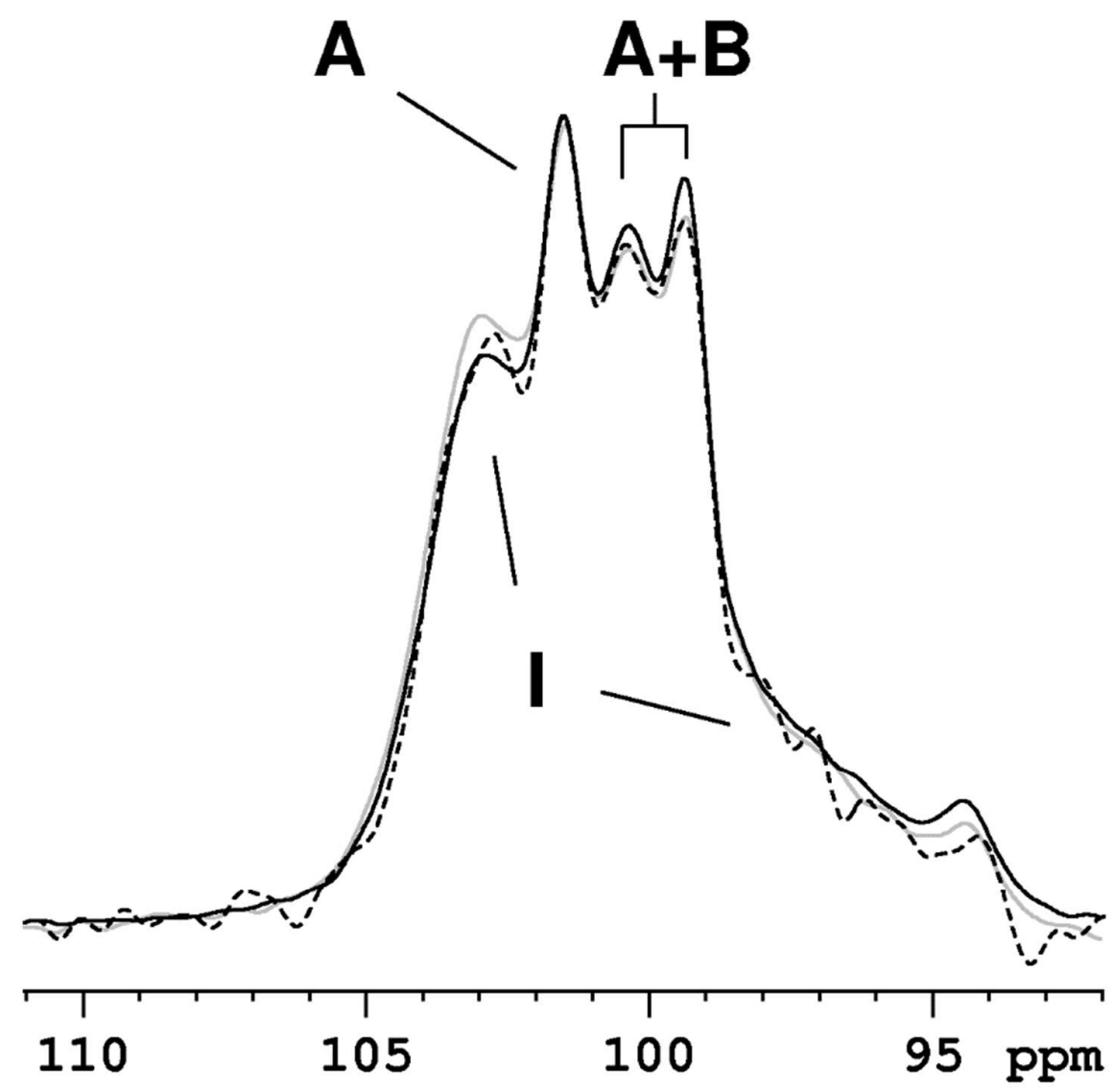

555

556

557

558

559

560

561

Figure 6 - Expansion of the CP-MAS ${ }^{13} \mathrm{C}-\mathrm{NMR}$ room temperature spectra (amylose $\mathrm{C}_{1}$ resonance region) of flours obtained from O. glaberrima (grey), O. sativa (dashed black) and NERICA (solid black). Resonance attributable to either A-type (A) or B-type (B) starch, or to "interfacial" carbons (I), are indicated. 


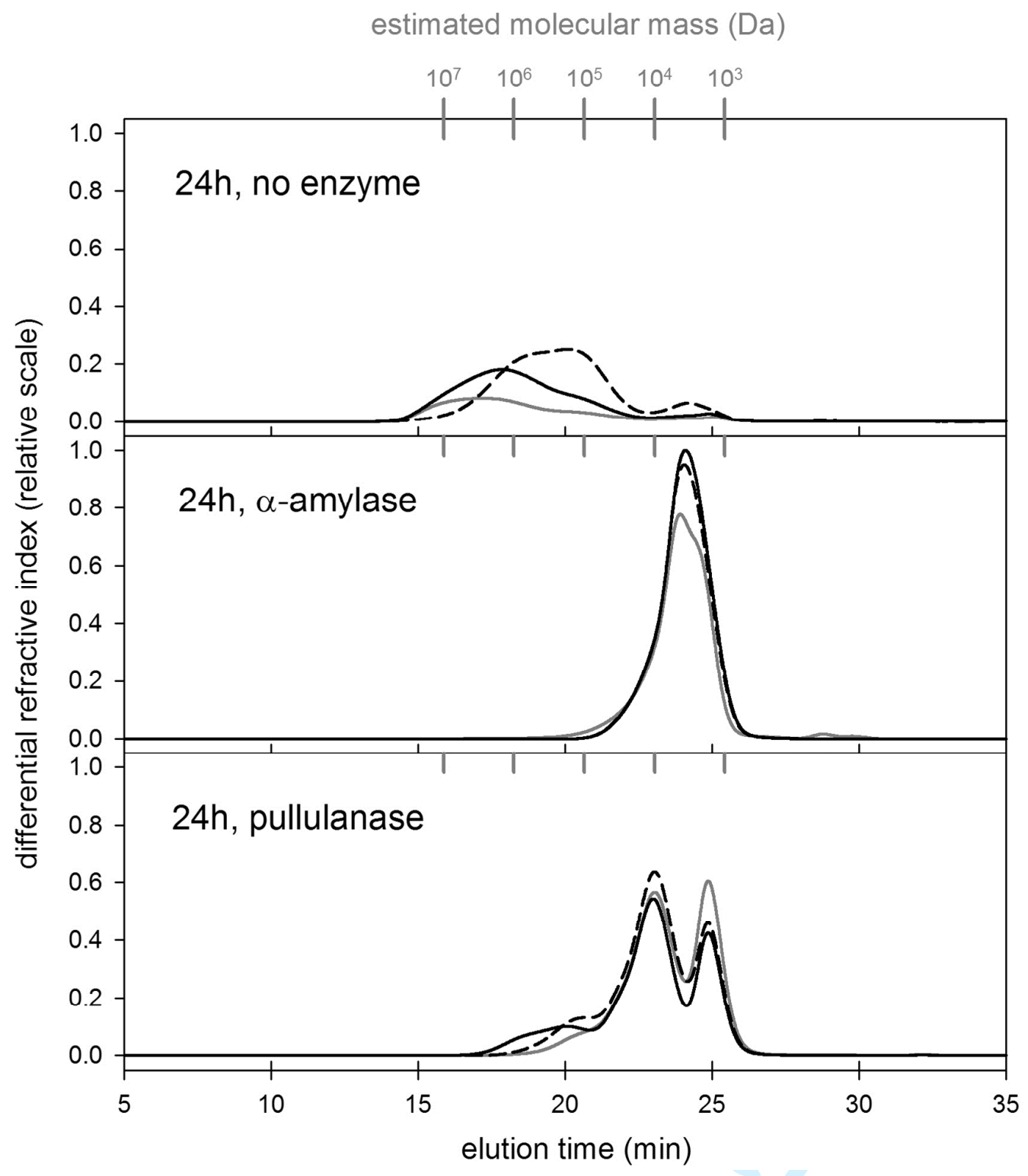

Figure 7 - SEC-HPLC profiles of soluble polysaccharides present in gelatinized starch from various rice grains after incubation at $37{ }^{\circ} \mathrm{C}$ in the absence/presence of different hydrolytic enzymes. The estimated molecular size of soluble molecules was derived from online Static Light Scattering (MALS) measurements. Grey, O. glaberrima; dashed black, O. sativa; solid black, NERICA. 
Dear Dr. Youling Xiong,

This accompanies a revised version (JFDS-2017-0564.R1) of the manuscript "Macromolecular traits in the African rice Oryza glaberrima and in glaberrima/sativa hybrids, and their relevance to processing".

We are grateful to both Reviewers for their positive attitude towards our work. We took into account all the remarks made by the Reviewers, and revised the text whenever appropriate. Aside from typos and language issues, that have been duly taken care of as indicated, here below are a couple of points where action was taken in response to specific points brought forward by Reviewer 2.

Q. Authors suggest that $O$. sativa traits may be responsible for controlling the effect of DTT on amount od proteins extracted. Could authors mention examples of such traits?

R. A sentence has been added to lines 236-240 of the revised manuscript to bring forward some evidence we had gathered on other cereals with respect to these issues, and appropriate references are also provided.

Q. Line 317-320 should be rephrased to better establish the flow of events

A: $\quad$ We cannot but concur that the construction of the original sentence was confusing at least. Following the Reviewers suggestion, we are now listing the various steps of this approach in temporal order and as separate sentences.

As requested, all changes made in the text are highlighted in red characters, including the newly added references.

Looking forward to hearing from you in due course, I remain,

Yours sincerely,

Stefania lametti, PhD

Professor of Biochemistry

University of Milan 